\title{
Adjustment of Tele-Operator Learning When Provided with Different Levels of Sensor Support While Driving Mobile Robots
}

\author{
David Sanders \\ School of Engineering \\ Portsmouth University \\ United Kingdom \\ david.sanders@port.ac.uk \\ David Ndzi \\ School of Engineering \\ Portsmouth University \\ United Kingdom \\ david.ndzi@port.ac.uk
}

\author{
Simon Chester \\ Chester Associates \\ Portsmouth \\ United Kingdom \\ simon.chester@simonchester.com \\ ManishMalik \\ School of Engineering \\ Portsmouth University \\ United Kingdom \\ manish.malik@port.ac.uk
}

\begin{abstract}
A quantitative and qualitative empirical evaluation is presented to show the effect of providing some sensor support during driving lessons as a tele-operator learns to drive a mobile robot. Different levels of sensor support were provided and the effect on training was measured. Different levels of force feedback were provided through a joystick. Results are presented and conclusions drawn about the way that teleoperators behave during driving tasks.
\end{abstract}

Keywords-Learning Mobile Robot Sensor; Tele-operation Ultrasonic

\section{INTRODUCTION}

Man-machine interfaces are becoming more important [1]. Research in tele-operation is attempting to improve safety and performance for tasks that cannot be fully automated and the distribution of tasks between humans and machines is a key issue to be considered [2].

Although 'Human-centered Automation' has become accepted as a principle for creating dependable man-machine systems, achievement is more difficult in actual circumstances [3]. Whatever a suitable amount of automation is, is influenced by many thing: expertise, intellectual work load; usefulness and reliability of the automated part of the system; and the amount of trust that an operator has in it [1]. Problems related to ongoing management of the automatic systems can outweigh any performance improvements.

Kirlik explored sensor and human operator interaction. He examined automated systems to study why aids are sometimes not used [4]. The importance of adjusting levels of automation to fit a changing situation was described in [5]. It was reported in recent work that time to carry out tele-operated tasks with mobile robots depends partly on the way in which human operators work together and cooperate with their robots [6], [7]. More work stated that tele-operators are likely to make use of visual feedback if available [8], [9] and the quantity of provided sensor support should be adjusted to suit the situation $[10,11]$.

Research described here examines how users adapt behaviour when using different levels of support. Results are employed to assess the outcome of offering assistance as teleoperator drivers learnt and developed skills. This work examined how tele-operators ought to be taught if various levels of support were accessible. Recent research suggested that sometimes, a skilled operator trained to operate a mobile robot base without sensors to help them then performed better when sensors were introduced to assist them [6]-[11]. A question considered here was, could a skilled operator trained with sensors to help them, then handle situations without sensors to assist? The way that tele-operators adapted their behaviour was investigated as they were provided with different levels of support. They behaved in different ways when encountering different conditions.

During traditional research to investigate using various levels of support, the participants have usually been experts with pre-developed skills. Acquiring skill has usually been ignored [1].

Two groups of experiments are presented. Comparable investigations with robot arms are described in [12], [13]. In the work described in this paper, constraint-based support that used virtual force feedback was used [1]. Force feedback represented the closeness to a wall or obstacle and this was similar to work by Volpe who represented force on a slave arm back at a joystick [14]. Constraint-based support provided a force back at the controller in order to evade collisions and direct an operator in a safer direction.

Part II of the paper describes the mobile robot system, and Part III describes the experiments. Part IV is some discussion and conclusions. 


\section{THE MOBILE RobOT SYSTEM}

A Bob Cat 2 base was modified to incorporate sensors and interfacing. The base had $2 \times$ main driving wheels forward and castors trailing in the rear. Ultrasonic sensors were fitted over each driving wheel. Changing the speed of rotation for each drive wheel changed the direction of the mobile robot.

Sonar sensors have been widely used in mobile-robotics [15]. Ultrasonics were chosen because they are simple and robust. Ultrasonics were transformed into a basic representation of the world ahead. Signals from the joystick were integrated if force-feedback was used so that a human tele-operator could override any propensity to rotate if close to an object, for example if the human driver wanted to touch or push an object.

Some algorithms to mix the joystick and sensor inputs were described in [16]. The mobile-robot was guided by computer as a "fly-by-wire" system. Links between the joystick and the mobile-robot were disconnected and instead, a computer combined inputs from the joystick and sensors and modified the direction and speed of the mobile-robot if needed. There was still an option for the joystick to directly control the mobile-robot. The programs were written using the methodology described in [17]-[23]. There were 3 principal levels: supervisory, strategic, servo-control. That was similar to [24]-[26]. The supervisory systems are explained in [27].

The following rules were applied by the programs:

- Tele-operator to be in control.

- Direction or speed of the mobile-robot only changed if essential.

- Movement to be controlled and smoothed.

The sensors provided knowledge about obstacles ahead of the mobile robot and the computer created a fictional potentialfield around them to guide the robot away from obstacles if there was a risk of collision.

\section{EXPERIMENTS}

Tele-operators were provided with one of three possible levels of support:

Level Zero (Lev0): No sensors to assist. No force feedback. The tele-operator could steer their robot without being interfered with. Risk of collision was higher but operators had more freedom of action.

Level One (Lev1): Sensors were engaged and thejoystick transmitted a repulsive force when the mobile robot was driven close to objects in its path. The size of repelling force was increased as distance to an object reduced.

Level Two (Lev2): Sensors were on and the mobile robot was automatically steered away from obstacles. Human teleoperators did not sense any resistive force if they moved the mobile robot close to an obstacle. The mobile robot was automatically steered away.

Level Three (Lev3): Both of Lev1 and Lev2 were delivered at the same time. Operators would be most constrained in this case.
The tele-operator could always force the robot to move against the applied force if they wanted to.

Number of collisions (C) and average times to complete tasks (T) were recorded and made use of as performance measures.

\section{A. Initial experiments}

Initial experiments compared Lev0 (sensors off) and Lev3 (sensors on, a repulsive force provided and automatic obstacle avoidance). More experiments compared Lev0, Lev1 and Lev2 with different environments.

Approval was obtained from the University Faculty ethics committee and participants gave their informed consent.

Participants drove a Bobcat II Mobile Robot Base [7, 10] through one of four courses. As an example, Fig 1 shows the first route used for the first set of experiments. That was a simple track from some doors, down the length of a corridor around three obstacles before driving around a corner to the finish. Obstacles are marked in black.

Courses were different lengths and included various obstacles.

Participants drove the mobile robot through each course and attempted to avoid obstacles. They used a joystick to drive the mobile robot. It was connected to the robot via an umbilical-cord so that they could observe the mobile robot directly throughout the tests.

A Forcefeedback Pro joystick from Microsoft was used to steer the mobile robot. It had force feedback built in and had been designed and used for games. It was unable to apply force quantitatively, but it had already been successfully used as a force feedback mechanism in some experimental studies at Tohoku University in Japan [1] and so was selected for this work.

For Lev3 tests, the resistive force increased if the teleoperator steered the robot close to an obstacle. Sensor support was more restrictive than Lev1 or Lev2 alone.

Participants were sixty staff and students (with no prior experience). They were separated in to two groups (A \& B). They were further sub- divided between the 4 courses; roughly eight participants within each sub-group.

Participants were shown the route. Then they drove the route 10 times each with and without sensors to assist. After that, A and B performed the tests with different levels of support. A second set of tests examined the performance of the tele-operators when they were confronted with different support conditions (this was after a participant had acquired some driving skill). Subjective information concerning preference was obtained using a simple questionnaire. Questions were:

"Do you prefer Lev0 or Lev3?"

"Which is easier to drive; Lev0 or Lev3?"

Table 1 shows the responses. 


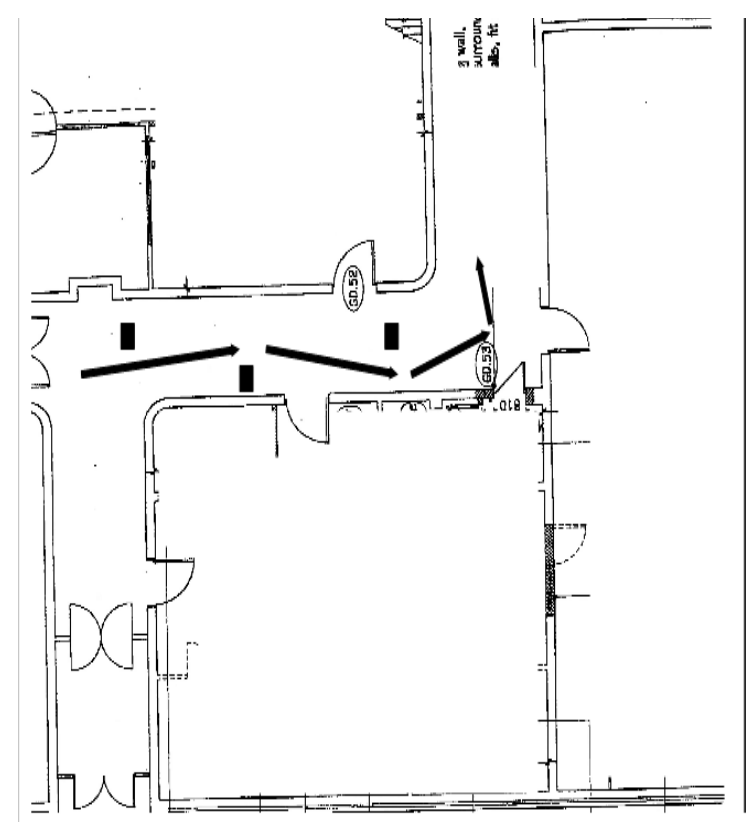

Fig. 1. First simple course used for testing.

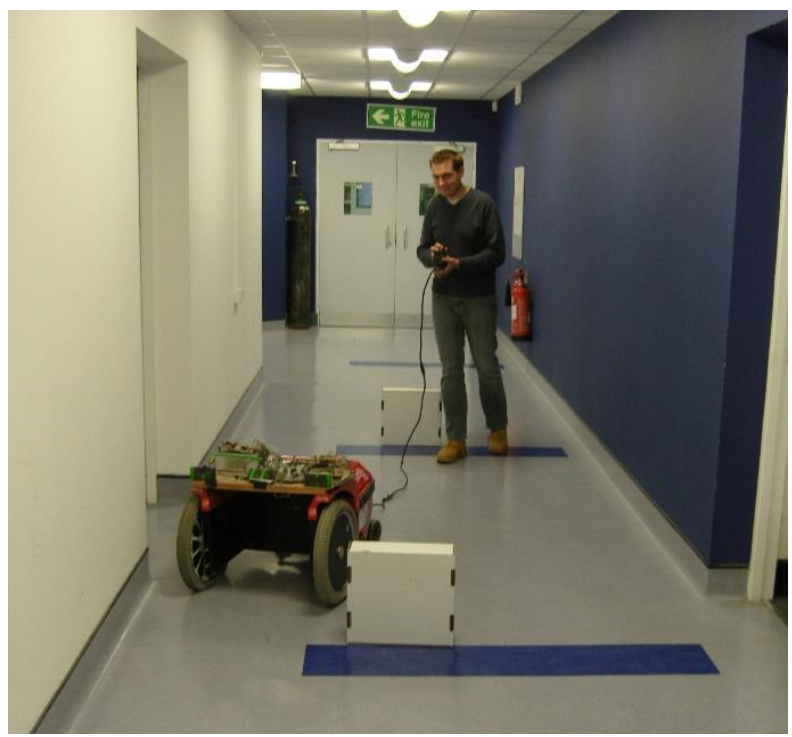

Fig. 2. Picture of part of Route Two with a participant driving a mobile robot.

TABLE I. Results From SubJective APPRAISAL (OBTAINED By QUESTIONNAIRE)

\begin{tabular}{|l|c|c|c|c|c|}
\hline & $\begin{array}{l}\text { Strongly } \\
\text { prefer } \\
\text { Level 0 }\end{array}$ & $\begin{array}{l}\text { Prefer } \\
\text { Level 0 }\end{array}$ & Undecided & $\begin{array}{l}\text { Prefer } \\
\text { Level 3 }\end{array}$ & $\begin{array}{l}\text { Strongly } \\
\text { prefer } \\
\text { Level 3 }\end{array}$ \\
\hline Preference & 13 & 15 & 17 & 12 & 3 \\
\hline How Easy? & 14 & 17 & 8 & 15 & 6 \\
\hline
\end{tabular}

\section{B. Results from initial experiments}

Average times in seconds for A and B for driving attempts in each of the four courses are shown in Figs $3 a$ and $3 b$.

Attempts one to ten on the horizontal axis correspond to the initial set of tests (A with Lev3 support and B with Lev0) and eleven to twenty correspond to the later experiments (A with Lev0 and B with Lev3).

Figure $3 \mathrm{a}$ and $3 \mathrm{~b}$ show that volunteer teleoperators in $\mathrm{A}$ (with force feedback) achieved a learning equilibrium sooner and more steadily than B. That suggests a helpful effect from using Lev3 during the initial learning when skill was being developed. Teleoperators in A also performed better during the second part of the tests, with Lev0 support. Skill acquisition was accelerated using Lev3 support.

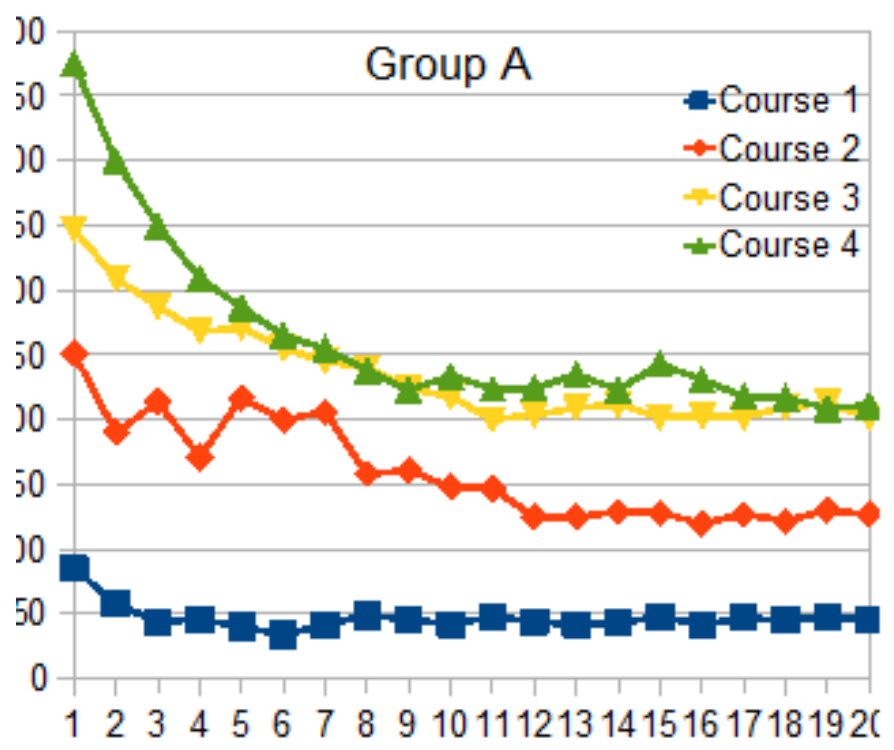

Fig.3.a: Lev3 support first, then Lev0 (Ultrasonic sensor system switched off). Average time $\mathrm{T}$ for $\mathrm{A}$ and $\mathrm{B}$ to drive through the four set courses.

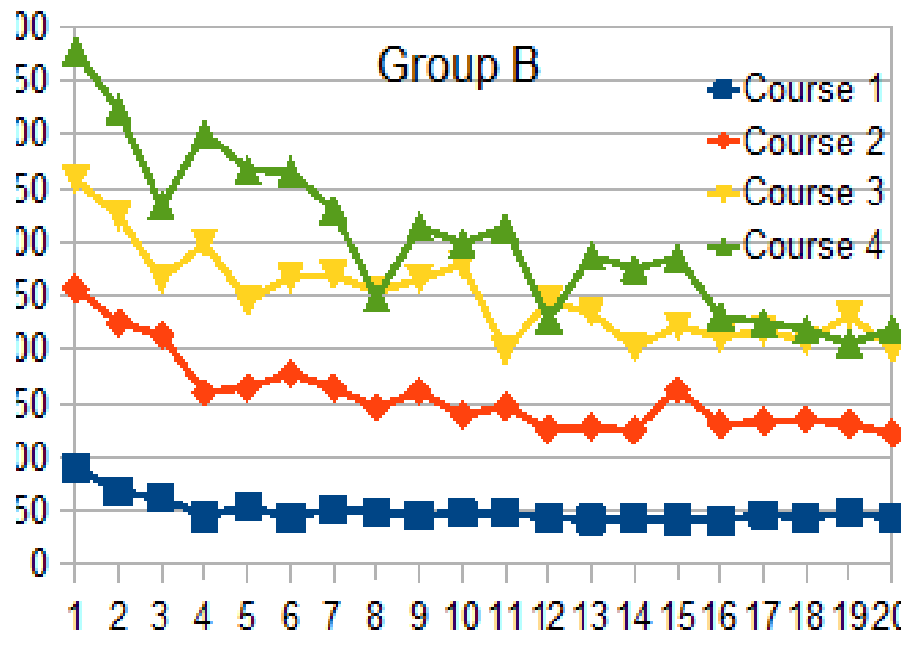

Fig.3.b: Lev0 support first then Lev3 (Lev1 and 2 provided simultaneously).

Fig. 3. Average time for A and B to complete four different courses 
Teleoperators may have attained general skill that could then be applied to new conditions (in this case operating the robot without any support).

Teleoperators in B did not show improvement in the second half of the trials (in terms of time taken and stability). That behaviour might be because skill developed with Lev0 support dos not transfer so easily to new situations or conditions.

Table 1 shows the results from subjective appraisal from the questionnaire. Participants suggested it was easier to drive without any sensor support (Lev0). That challenged the objective findings. Subjective answers also said constraintbased support was disliked.

\section{Discussion of the first set of experiments}

Initial tests suggested a tele-operator who trained with sensors to assist them still handled situations without sensors to assist (when removed). I addition, support from sensors during learning had positive effects on later implementation when operators did not have support.

Some subjects may have become used to steering the robot through the same paths throughout the tests. More tests are needed to check that skill can transfer to new working conditions when there is not any support but results do agree with Chikura [1].

Chikura believed a tele-operator taught without any support would not perform better when using sensors to assist them.

Results also indicated that teleoperators trained without support didn't demonstrate a steady learning curve when compared with teleoperators who did not have previous experience. Tele-operator skill developed and learned when driving without assistance had negative effects on performance when assistance was provided later. That is important for training procedures for intelligent systems such as [6].

Informal discussion revealed that was partially as they teloperators felt their freedom of decision making and movement was being constrained. That contradiction between objective execution and the self-biased comments indicated that decisions about training based on student preference could lead to poorer implementation.

\section{Abbreviations and AcronymsSecond set of experiments}

The second set of experiments investigated the way that behaviour adapted if working conditions changed and participants had new levels of support.

Later experiments used a different route. It was more complicated and longer (Fig 4). Obstacles could be repositioned to create separate routes along the same path.

These experiments were to examine differences in behaviour with varying amounts of support from the sensors when new conditions were encountered.

Each participant completed the route six times before the route was changed by repositioning obstacles. Then the participants completed a second and third set of six attempts on another two new and altered courses.

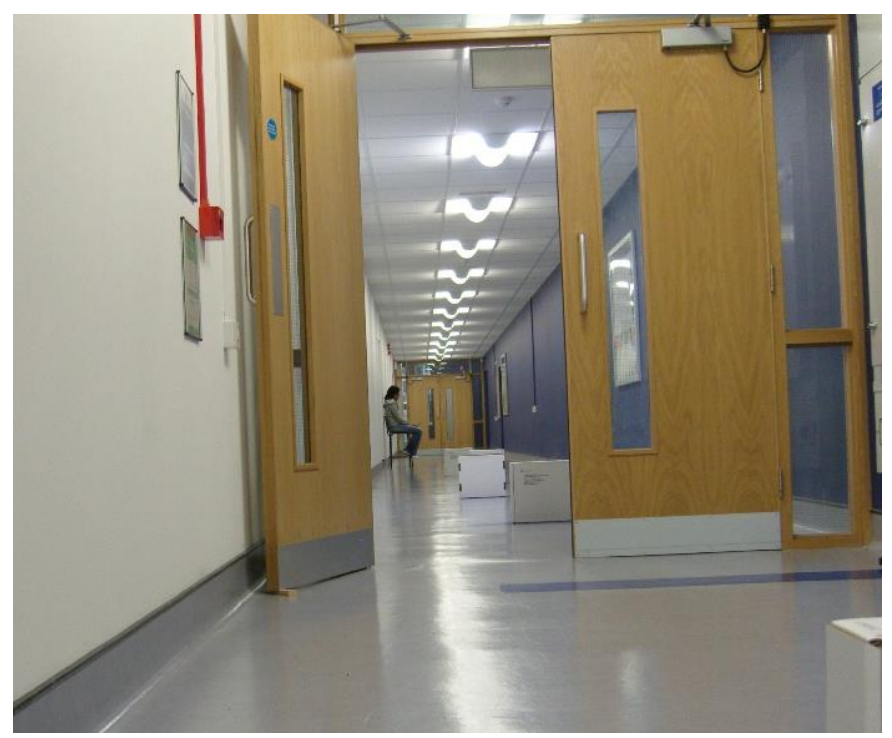

Fig. 4. Part of the course used for the phase two study.

Each tele-operator performed each test with the identical support settings for all the three sets of six.

Eleven participants who had not taken art in the initial tests, were split into three groups (Group X, Group Y, Group Z). X completed tasks with lev0 (controlled manually) and $\mathrm{Y}$ with lev1 (a repulsive force produced) and $\mathrm{Z}$ with Lev2 (automatically steering the mobile robot around objects).

Time taken to compete courses was recorded along with the number of collisions.

\section{E. Results from later experiments}

Fig 5 (a), 5(b), and 5(c) show the time taken in seconds for the second set of trials for Group X, Group Y and Group Z.

Lev3 support resulted in the smallest amount of collisions although completion time became worse compared with Lev0 support.

Differences in the completion times when the layout of the course (position of obstacles etc) was adjusted was assessed. Completion time tended to increase for Lev1 and lev2, while completion time did not change for Lev0.

This could suggest that skills learned without sensors supporting the operator could be more generic when compared to skills learned with sensor support.

The number of collisions is shown in Fig 6 for each volunteer.

\section{F. Discussion concerning later experiments}

There appeared to be differences in behavior and adaptation for different support levels.

$\mathrm{T}$ showed different behaviour for each level when the course was changed 


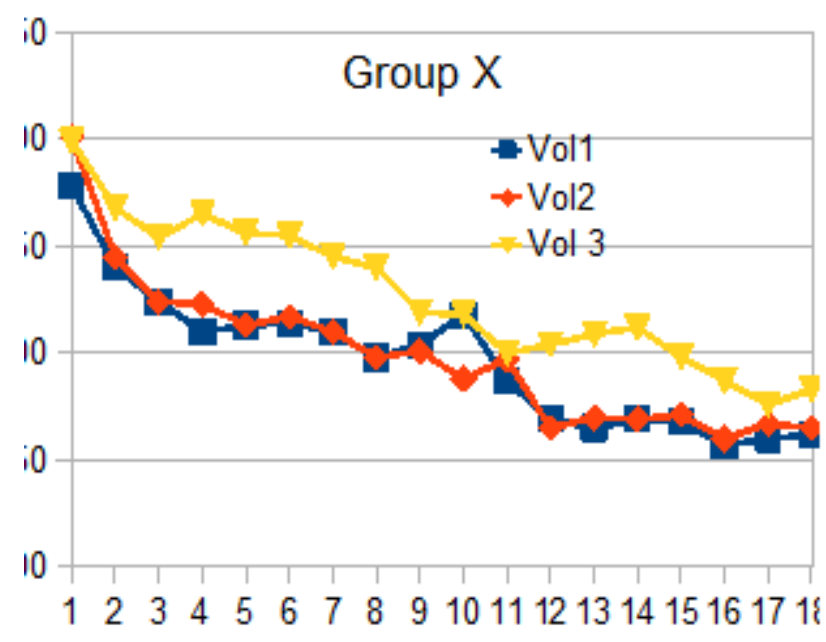

Fig. 5. a: Lev0 - Ultrasonic sensor system switched off

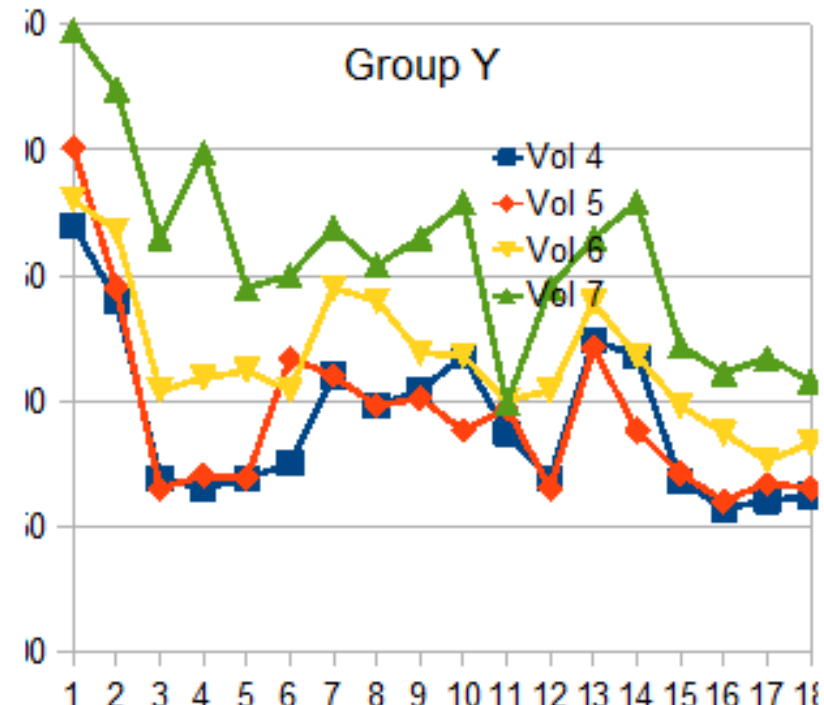

Fig. 5. b: Lev1 - Sensor system switched on and repulsive force provided

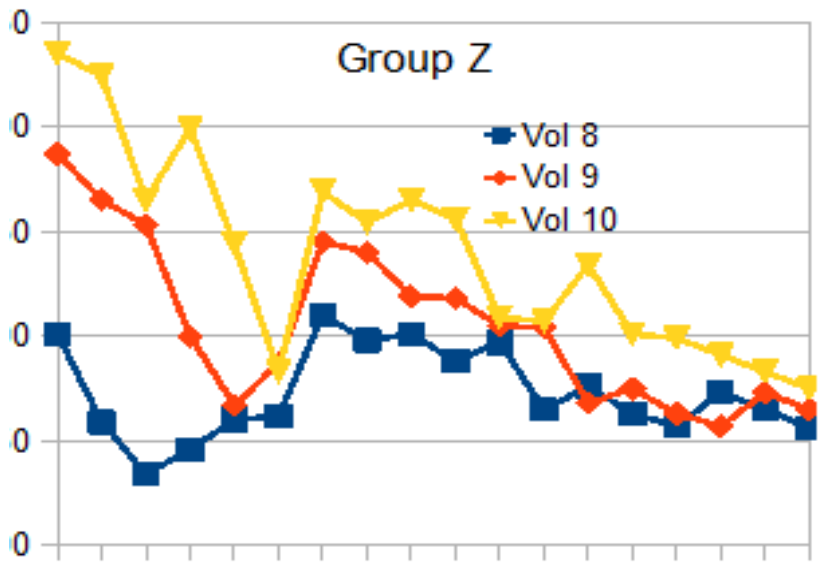

$\begin{array}{lllllllllll}1 & 2 & 3 & 4 & 5 & 6 & 7 & 8 & 9 & 10111213141516171 \text { \& }\end{array}$

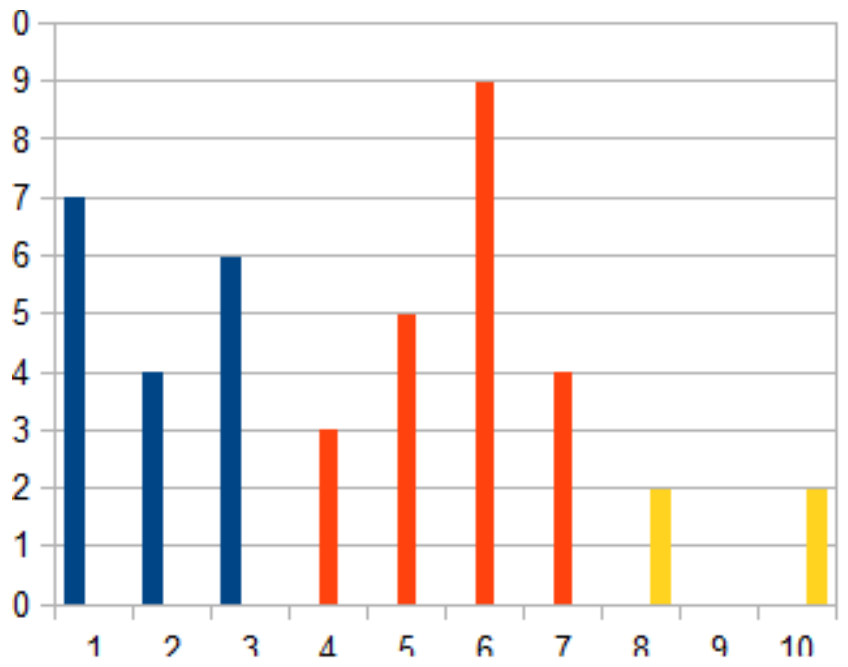

Fig. 6. Number of collisions for each tele-operator

Fig. 7.

\section{OVERALl DiSCUSSION AND CONCLUSIONS}

Behaviour with a variety of amounts of support was investigated to see whether using sensors assisted during teaching.

Using sensor systems to assist had a positive effect. Learning while driving manually and then using sensor systems had a negative effect. In later experiments, Lev2 decreased collisions.

More participants could have improved the accuracy of results during later experiments. Additionally, the results from later experiments are inconsistent compared with results obtained during initial testing.

The initial tests suggested that support during learning helped when compared with driving manually. That was not the case for the later experiments.

Further tests are required to consider the performance of individual participants. Results presented here provide an insight into behaviour of tele-operators learning in different conditions and situations and with varying levels of support.

Although this paper suggests that using sensors to assist during training is effective, some other work has said that proficient users perform better in unrestricted environments without any sensors to assist [7-9]. In both cases though, the assistive systems are more and more helpful as environments become more and more complex or if the view of the mobile robot is limited (or the communications and / or control signals are delayed).

Work is now investigating mixing other AI tools [28-37] to use specific tools where they can have most effect.

Fig. 5. c: Lev2 - System automatically steered the mobile robot away from obstacles 


\section{REFERENCES}

[1] D Chikura, M Takahashi, S Watanabe and M Kitamura, Adaptation of User Behavior to the Different Level of Tele-Operation Support:. IEEE International Conference on Systems, Man, and Cybernetics. Volume 3, pp: 739 - 744. 1999

[2] Sheridan TB, Telerobotics, Automalion and Human Supervisory Control, Cambridge Massachusetts: The MIT Press, 1992.

[3] Sheridan TB, "Human-centered automalion: oxymoron or common sense? ," Proc. IEEE International Conference on Systems, Man and Cybernetics, Vancouver Canada, 1996.

[4] Kirlik A, "Modeling Behavior in Human-Automation Interaction: Why an "Aid Can(and Should) Go Unused," Human Factors, 35(2),pp.22 1242. 1993

[5] Itoh Met. al, "Experimental study of" situation-adaptive humanautomation collaboration for takeoff safety, Proc. of the 7th IFAC/IFIP/IFORS/IEA Symposium on Analysis, Design and Evaluation of Man-Machine Systems, pp.37 1-376. 1998.

[6] Sanders DA; Graham-Jones J and Gegov A. Improving ability of teleoperators to complete progressively more difficult mobile robot paths using simple expert systems and ultrasonic sensors. Industrial Robot Volume: 37, Issue: 5, Pages: 431-440. 2010

[7] Sanders D. Analysis of the effects of time delays on the teleoperation of a mobile robot in various modes of operation. Industrial Robot Volume: 36, Issue: 6, Pages: 570-584. 2009

[8] Sanders D; Tewkesbury G; Stott IJ.; et al. Simple expert systems to improve an ultrasonic sensor-system for a tele-operated mobile-robot. Sensor Review 31 (3), pp: 246-260. 2011

[9] Sanders, D. Comparing ability to complete simple tele-operated rescue or maintenance mobile-robot tasks with and without a sensor system. Sensor Review 30 (1), pp: 40-50. 2010

[10] Sanders, D. Controlling the direction of "walkie" type forklifts and pallet jacks on sloping ground. Assembly Automation Volume: 28, Issue: 4, Pages: 317-324. 2008

[11] Sanders DA, Stott I, Robinson D and Ndzi D. Analysis of successes and failures with a tele-operated mobile robot in various modes of operation. Robotica 30, pp 973-988. 2012.

[12] Backes PG, "Supervised Autonomy for Space Robotics," Progress in Astronautics and Aeronautics 161, pp. 139-158. 1994

[13] Draper JV et.al. "Measuring Operator Skill and Teleoprator Performance," Proc. of International Symposium on Teleoperation and Contro1,1998.

[14] Volpe R, "Techniques for Collision Prevention ,Impact Stability, and Force Control by Space Robots", Progress in Astronautics and Aeronautics, Vol.161, 1994, pp.I75-212.

[15] Gao W and Hinders M. Mobile robot sonar backscatter algorithm for automatically distinguishing walls, fences, and hedges. Int Jnl of Robotics Research 25 (2), pp 135-145. 2006.

[16] Stott IJ, Sanders DA and Goodwin MJ. A software algorithm for the intelligent mixing of inputs to a tele-operated vehicle. Jnl of Systems Architecture 43 (1-5), pp 67-72. 1997.

[17] Chester, S; Tewkesbury, G; Sanders, D, et al. New electronic multimedia assessment system. Web Information Systems and Technologies 1, pp 414-420. 2007.

[18] Chester, S; Tewkesbury, G; Sanders, D, et al. New electronic multimedia assessment system. WEBIST 2006: Proceedings of the Second International Conference on Web Information Systems and Technologies, pp 320-324. 2006.

[19] Bergasa-Suso, J; Sanders, DA and Tewkesbury, GE. Intelligent browser-based systems to assist Internet users. IEEE Transactions on Education 48 (4), pp 580-585. 2005.

[20] Sanders DA, Cawte H, Hudson AD. Modelling of the fluid dynamic processes in a high-recirculation airlift reactor. Int Jnl of Energy Research 25 (6), pp 487-500. 2001.

[21] Sanders, DA and Rasol, Z. An automatic system for simple spot welding tasks. Total Vehicle Technology: Challenging current thinking, pp 263-272. 2001.
[22] Sanders, DA and Hudson, AD. A specific blackboard expert system to simulate and automate the design of high recirculation airlift reactors. Mathematics and computers in simulation 53 (1-2), pp 41-65. 2000.

[23] Sanders, DA; Hudson, AD; Tewkesbury, GE, et al. Automating the design of high-recirculation airlift reactors using a blackboard framework. Expert syst with applications 18 (3), pp 231-245. 2000.

[24] Tewkesbury GE and Sanders DA. A new simulation based robot command library applied to three robots. Jnl of Robotic Systems 16 (8), pp 461-469. 1999.

[25] Sanders DA. "Using self-reliance factors to decide how to share control between human powered wheelchair drivers and ultrasonic sensors" IEEE Transactions on Neural Systems and Rehabilitation Engineering, In Press. DOI: 10.1109/TNSRE.2016.2620988. 2016.

[26] Tewkesbury GE and Sanders DA. A new robot command library which includes simulation. Jnl of Robotic Systems 26 (1), pp 39-48. 1999.

[27] Sanders DA. Comparing speed to complete progressively more difficult mobile robot paths between human tele-operators and humans with sensor-systems to assist, Assembly Automation 29 (3), pp 230-248. 2009.

[28] D. Sanders and A. Geov. "AI tools for use in Assem. Autom. and some examples of recent applications. Assem. Autom. Vol 33 (2), pp: 184194. 2013.

[29] D. A. Sanders, G. E. Tewkesbury, and D. Ndzi et al. "Improving automatic robotic welding in shipbuilding through the introduction of a corner-finding algorithm to help recognise shipbuilding parts". Journal of Marine Science and Technology Vol 17 (2), pp: 231-238. 2012.

[30] D. Sanders, G. Lambert, and J. Graham-Jones et al. "A robotic welding system using image processing techniques and a CAD model to provide information to a multi-intelligent decision module". Assem. Autom. Vol: 30 (4), pp: 323-332. 2010.

[31] D. A. Sanders, and G. E. Tewkesbury. "A pointer device for TFT display screens that determines position by detecting colours on the display using a colour sensor and an Artificial Neural Network". Displays Vol 30 (2), pp: 84-96. 2009.

[32] J. Bergasa-Suso, D. A. Sanders, and G. E. Tewkesbury. "Intelligent browser-based systems to assist Internet users". IEEE Transactions on Education Vol 48 (4), pp: 580-585. 2005.

[33] D. A. Sanders, Y. Tan, I Rogers et al. "An expert system for automatic design-for-assembly”. Assem. Autom. 29 (4), pp: 378-388. 2009.

[34] D. A. Sanders, G. Lambert, and L. Pevy. "Pre-locating corners in images in order to improve the extraction of Fourier descriptors and subsequent recognition of shipbuilding parts". Proc of IMechE Part BJournal of Engineering Manufacture 223 (9), pp: 1217-1223. 2009.

[35] D. Sanders. "Controlling the direction of walkie type forklifts and pallet jacks on sloping ground". Assem. Autom. 28 (4), pp: 317-324. 2008.

[36] D. A. Sanders. "Progress in machine intelligence". Ind Rob: An int' jnl 35 (6), pp: 485-487. 2008.

[37] A. Geov, N. Gobalakrishnan and D. A. Sanders. "Rule base compression in fuzzy systems by filtration of non-monotonic rules". Journal of Intelligent \& Fuzzy Systems Vol 27 (4), pp: 2029-2043. 2014. 